\title{
Resource Allocation for OFDM-NOMA Systems under Multiuser Scenarios with Different Speeds
}

\author{
Pengfei Wu ${ }^{D},{ }^{1}$ Wei Xiao, ${ }^{1}$ Zhicheng Dong $\left(D,{ }^{1}\right.$ Jingjing Cui, ${ }^{2}$ Fuhua An, ${ }^{1}$ Weixi Zhou, ${ }^{3}$ \\ Xiaoyu Li, ${ }^{4}$ and Fangkai $\mathrm{Cai}^{4}$ \\ ${ }^{1}$ Tibet University and School of Information Science and Technology, China \\ ${ }^{2}$ University of Southampton, School of Electronics and Computer Science, UK \\ ${ }^{3}$ Sichuan Normal University School of Computer Science, China \\ ${ }^{4}$ Chengdu Technological University, School of Network and Communication Engineering, China
}

Correspondence should be addressed to Zhicheng Dong; dongzc666@163.com

Received 6 November 2021; Revised 29 January 2022; Accepted 2 February 2022; Published 3 March 2022

Academic Editor: Issa Elfergani

Copyright (c) 2022 Pengfei Wu et al. This is an open access article distributed under the Creative Commons Attribution License, which permits unrestricted use, distribution, and reproduction in any medium, provided the original work is properly cited.

In this paper, we consider an OFDM-NOMA (orthogonal frequency division multiplexing-nonorthogonal multiple access) downlink system, which has multiple subcarriers and multiple users at different locations and speeds from the base station. The goal is to maximize energy efficiency (EE), which is the bit rate per unit of energy consumption, while meeting the user's quality of service and power constraints. Since the optimization problem of joint user subcarrier allocation and power allocation is a mixed-integer nonlinear programming (MINLP) problem, the use of traversal search will produce an unacceptable amount of calculation. It should be noted that the optimization problem includes the variables of user and subcarrier allocation and power allocation coefficient. In order to effectively solve this problem, we divide the problem into two subproblems: the problem of user and subcarrier allocation and the problem of power allocation between users. We use a matching algorithm to solve the problem of user and subcarrier assignment. An iterative algorithm to ensure convergence is proposed to obtain the power budget between subcarriers. Finally, the effective binary search is used to obtain the power allocation among users. The simulation results show that the proposed algorithm has a faster convergence rate. At the same time, as the number of users and subcarriers increases, EE improves significantly. As the user speed increases, the EE gradually decreases; especially when the number of users is larger, the effect is more obvious. Compared with random matching, the proposed algorithm has higher EE under the same number of users, number of subcarriers, and speed.

\section{Introduction}

The explosive growth of today's data traffic has brought enormous challenges to the limited spectrum resources. Nonorthogonal multiple access (NOMA) technology is widely used in $5 \mathrm{G}$ mobile networks as a spectrum efficient multiple access technology [1]. NOMA technology allows multiple users to share spectral resources in the same spatial layer in both time and power domains. Compared with orthogonal multiple access (OMA), NOMA can support a large number of connections and achieve higher spectral efficiency [2]. NOMA allows multiple users to access the same orthogonal resource blocks, such as frequency bands, time slots, and spatial directions. Interuser interference can be eliminated at the receiving end by using a sequential interference cancellation (SIC) receiver for multiuser detection [3].

Liang et al. [4] proposed user pairing based on one-toone matching and studied the problem of power allocation in cognitive radio NOMA systems. In addition, [5] proposed a subchannel allocation method for a downlink NOMA system using the many-to-many matching theory. In heterogeneous networks, a suboptimal algorithm to alternately optimize the resource allocation of macrocells and small cells is proposed in [6]. Zhang et al. [7] designed a lowcomplexity subcarrier matching algorithm by decoupling subchannel allocation and power control. In incomplete CSI downlink NOMA heterogeneous networks, Song et al. 
[8] transformed the probabilistic problem into a nonprobabilistic problem through relaxation. Xu et al. [9] used joint optimization problem control involving user association, computing resource allocation, and transmit power. A Dinkelbach-like algorithm [10] is employed, by which each individual fractional $\mathrm{EE}$ function is transformed into a parametric function. In assisted cognitive radio nonorthogonal multiple access (CR-NOMA) networks, Zhao et al. [11] proposed a multiobjective iterative algorithm (MOIA) to achieve a joint optimal solution.

Through the above research, we study the resource allocation of the OFDM-NOMA system in multiuser scenarios with different speeds. Since the optimization problem of joint user subcarrier allocation and power allocation is a mixed-integer nonlinear programming (MINLP) problem, the use of traversal search will produce an unacceptable amount of calculation; we divide the problem into two subproblems: the problem of user and subcarrier allocation and the problem of power allocation between users. The main contributions of this paper are as follows:

(1) This article considers the resource allocation of the OFDM-NOMA system in multiuser scenarios with different speeds. In order to solve the problem of grouped users and subcarriers, we adopted a matching algorithm. Firstly, users and suboperators establish their own preference lists and then perform matching exchanges through matching algorithms to obtain suboptimal solutions to solve the user grouping problem

(2) For the power allocation problem of different users on the same subcarrier, an iterative algorithm that guarantees convergence is proposed, which converts the nonconvex problem into a convex problem, obtains the power allocation for subcarriers, and then uses the effective binary search to solve the power allocation problem of different users on the same subcarrier

(3) The simulation results show that as the number of users and subcarriers increases, EE improves significantly. Compared with random matching, the algorithm proposed has higher energy efficiency (EE) under the same conditions

The remaining sections of this article are arranged as follows: In Section 2, we introduce system model and problem formulation. Resource allocations are reported in Section 3. Section 4 shows the simulation results and analysis. Finally, the conclusion is given in Section 5 .

\section{System Model and Problem Formulation}

2.1. System Model. Consider an OFDM-NOMA (orthogonal frequency division multiplexing-nonorthogonal multiple access) downlink system with $N$ subcarriers and $K$ active users with different speeds and distances from base station (BS). We assume that the users' velocities are also uniformly distributed. Different from the conventional OFDMA down- link system, a subcarrier can be shared by more than one user in the OFDM-NOMA system. The system model is shown in Figure 1. Let $\eta_{n, k} \in\{0,1\}$, where $\eta_{n, k}=1$ denotes that the $n$th subcarrier is assigned to the user $k, \eta_{n, k}=0$; if otherwise, then we can express the superposition symbol $X(n)$ on the subcarrier $n$ as

$$
X(n)=\sum_{k=1}^{K} \eta_{n, k} \sqrt{P_{n, k}} s_{n, k}
$$

where $P_{n, k}$ is the transmit power between the BS and the user $k$ on the subcarrier $n$ and $s_{n, k}$ denotes the unit energy $\mathbb{E}\left[\left|s_{n, k}\right|^{2}\right]=1$, where $\mathbb{E}$ represents the expectation operation.

The symbols $[X(1), \cdots, X(N)]$ are transformed into an OFDM symbol by inverse fast Fourier transformation (IFFT). A cyclic prefix (CP) is then added to eliminate the effect of the intersymbol interference (ISI). The time-domain transmitted signal can be written as $x(i)=$ $(1 / \sqrt{N}) \sum_{n=0}^{N-1} X(n) e^{(j 2 \pi i n / N)},-L_{\max } \leq i \leq N-1$, where $L_{\max }$ is the length of the guard interval for all users. The received signal $y_{k}(i)$ in time-domain at the time $i$ for user $k$ can be expressed as

$$
y_{k}(i)=\sum_{l=0}^{L} h_{k}(i, l) x(i-l)+\omega(i)
$$

where $h_{k}(i, l)$ is the channel impulse response of the $l$ th tap at time $i$ for the user $k$. We assume the Rayleigh fading channels, and a Jakes' Doppler spectrum with a maximum Doppler frequency $f_{k}^{\max }=f_{c} v_{k} / c$ for user $k$, where $f_{c}$ is the carrier frequency $(\mathrm{Hz}), v_{k}$ is the speed of the mobile terminal $(\mathrm{km} / \mathrm{h})$ for user $k$, and $c$ is the speed of light.

Therefore, the autocorrelation function of the timevarying channels can be expressed as $\mathbb{E}\left[h_{k}\left(i_{1}, l_{1}\right) h_{k}^{*}\left(i_{2}, l_{2}\right)\right]=$ $c_{1} e^{-l_{1} / L} J_{0}\left(2 \pi f_{c} v_{k} T\left(i_{2}-i_{1}\right) / c N\right) \delta\left(l_{1}-l_{2}\right)$, where $c_{1}$ is a constant, obtained to meet $c_{1} \sum_{l} e^{-l / L}=1 . J_{0}($.$) denotes the$ zeroth-order Bessel function of the first kind, and $T$ is the duration of an OFDM symbol. $\delta$ denotes the impulse function, and * stands for conjugate transposition. Assume that the maximum channel delay spread $L$ is less than or equal to the guard interval $L_{\max }$ for all users, and $\omega(i)$ is a zero-mean, complex additive white Gaussian noise with variance $\sigma_{\omega}^{2}$.

$$
Y_{k}(n)=\frac{1}{\sqrt{N}} \sum_{i=0}^{N-1} y_{k}(i) e^{-\frac{j 2 \pi n}{N}}=X(n) H_{n}^{k}+I_{n}^{k}+W_{n}^{k} .
$$

The second term in (3) denotes the intercarrier interference (ICI) caused by the time-varying nature of the channel. Since the data on each subcarrier are uncorrelated, the ICI power of the $n$th OFDM subcarrier for the user $k$ with the velocity $v_{k}$ can be presented as follows $[7,12,13]$.

$$
E\left\{\left|I_{n}^{k}\right|^{2}\right\}=\sum_{m=0, m \neq n}^{N-1} P_{m} \rho_{n, m}^{k}
$$




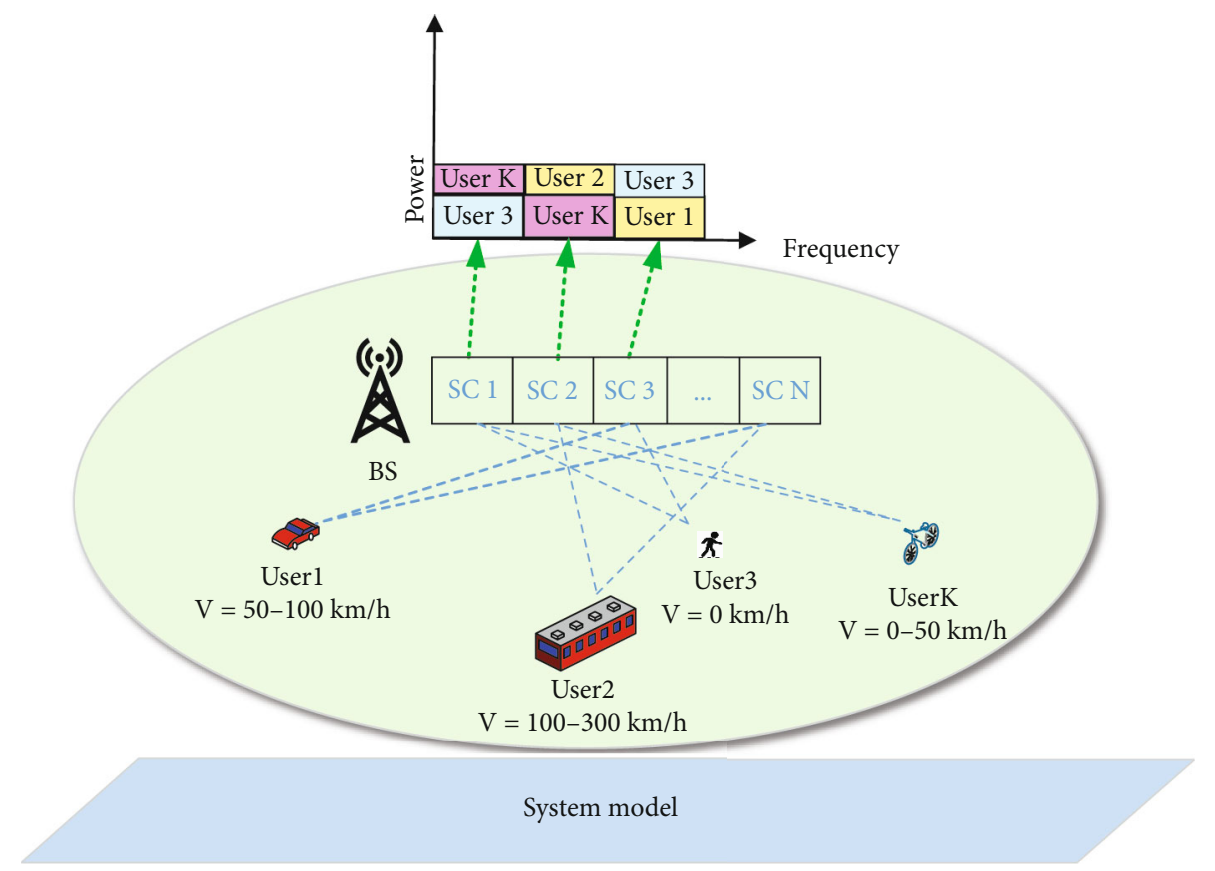

Figure 1: NOMA systems under multiuser scenarios with different speeds.

where $P_{m}=\sum_{k=1}^{K} \eta_{m, k} P_{m}^{k}$ is the total power consumption on the subcarrier $m$. $\rho_{n, m}^{k}=\left(1 / N^{2}\right)\left(N+2 \sum_{i=1}^{N-1}(N-i) J_{0}\right.$ $\left.\left(2 \pi f_{c} v T_{i} / c N\right) \cos (2 \pi i(n-m) / N)\right)$, and $J_{0}(\cdot)$ denotes the zeroth-order Bessel function of the first kind [14]. $T$ is the duration of an OFDM symbol. From (4), we can obtain that the ICI term monotone increases with increasing velocity. Then, $g_{n}^{k}$, regarded as an equivalent channel gain between the BS and the user $k$ on the subcarrier $n$ with the velocity $v_{k}$, can be expressed as follows [15]:

$$
g_{n}^{k}=\frac{\left|H_{n}^{k}\right|^{2} \eta_{n, k}}{\sum_{m=0, m \neq n}^{N-1} P_{m} \rho_{n, m}^{k}+\sum_{i=1, i \neq k}^{K}\left|H_{n}^{i}\right|^{2} P_{n}^{i}+\sigma_{\omega}^{2}} .
$$

The NOMA protocol is used in each subcarrier in this article. Specifically, consider a pair of two users $k$ and $j$ with different velocities $v_{k}$ and $v_{j}$, in which the user $k$ wants to decode and remove the user $j^{\prime}$ s signal by successive interference cancellation (SIC) at the receiver on the subcarrier $n$; then the following inequality holds: $g_{n}^{k} \geq g_{n}^{j}$. In the NOMA system, SIC can be implemented for the users with stronger equivalent channel gains. Generally, it is assumed that all the equivalent channel gains on the subcarrier $n$ follow the order as $g_{n}^{\pi(1)} \geq \cdots \geq g_{n}^{\pi(k)} \geq \cdots \geq g_{n}^{\pi(K)}$, where $\pi(k)$ presents the $k$ th decoded the users' index. Therefore, $g_{n}^{\pi(K)}$ first decodes the information of the users from $K$ to $k+1$ and then successively subtracts these information to obtain its own information. In the conventional NOMA system, the order is sorted by the channel gains resulting from different distances between the BS and the users [3]. However, the different velocities can also be used to sort the equivalent channel gains' order in this article. Therefore, based on the analysis of (4), users with slower velocities may guarantee that the equivalent channel gain is better than that of faster velocities' users.

Based on the above, the received signal to interference plus noise ratio (SINR) for the user $\pi(k)$ at the subcarrier $n$ with the velocity $v_{k}$ is given in (6).

$$
r_{n}^{\pi(k)}=\frac{\left|H_{n}^{\pi(k)}\right|^{2} \eta_{n, \pi(k)} P_{n}^{\pi(k)}}{\sum_{i=1}^{k-1}\left|H_{n}^{\pi(k)}\right|^{2} \eta_{n, \pi(i)} P_{n}^{\pi(i)}+\sum_{m=0, m \neq n}^{N-1} P_{m} \rho_{n, m}^{\pi(k)}+\sigma_{\omega}^{2}} .
$$

The SINR performance on the subcarrier $n$ for the user $k$ is impacted not only by noise and ICI but also by multiuser interference from SIC if the users have stronger equivalent channel gains than that of the $k$ th decode user. Therefore, we can obtain the achievable rate of user $\pi(k)$ at the subcarrier $n$ with velocity $v_{k}$, which is given by (7).

$$
C_{n}^{\pi(k)}=\frac{B}{N} \log \left(1+r_{n}^{\pi(k)}\right) .
$$

We define the total EE as the number of successfully transmitted bits per energy unit (bit/joule), or equivalently, the data rate per power unit (bps/watt). Therefore, we obtain the total EE of the small cell tier networks as

$$
\begin{aligned}
\varphi & =\frac{R_{t o t}}{P_{t o t}}, \\
R_{t o t} & =\sum_{k=1}^{K} \sum_{n=1}^{N} C_{n}^{\pi(k)}, \\
P_{t o t} & =\sum_{k=1}^{K} \sum_{n=1}^{N} P_{n}^{k}+P_{c},
\end{aligned}
$$

where $P_{c}$ is the circuit power consumption. 
2.2. Problem Formulation EE. We formulate the problem to optimize the problem based on user, subcarrier assignment, and power allocation. The optimization problem can be expressed as follows:

$$
\max _{\left\{P_{n}^{\pi(k)}\right\},\left\{\eta_{n, \pi(k)}\right\}} \varphi\left(P_{n}^{\pi(k)}, \eta_{n}^{\pi(k)}\right)=\frac{R_{\text {tot }}\left(P_{n}^{\pi(k)}, \eta_{n}^{\pi(k)}\right)}{P_{t o t}\left(P_{n}^{\pi(k)}, \eta_{n}^{\pi(k)}\right)},
$$

such that

$$
\begin{aligned}
& C 1: g_{n}^{\pi(k)} \leq g_{n}^{\pi(j)}, \pi(k) \geq \pi(j), \forall n, k, \\
& C 2: \sum_{k=1}^{K} \sum_{n=1}^{N} P_{n}^{\pi(k)} \eta_{n, \pi(k)} \leq P_{\max }, \forall n, k, \\
& C 3: C_{n}^{\pi(k)} \geq R_{t h}, \forall n, k, \\
& C 4: \sum_{k=1}^{K} \eta_{n, \pi(k)} \leq K_{f}, \\
& C 5: \sum_{n=1}^{N} \eta_{n, \pi(k)} \leq N_{f}, \\
& C 6: \eta_{n, \pi(k)}=\{0,1\}, \forall n, k, \\
& C 7: P_{n}^{\pi(k)} \geq 0, \forall n, k,
\end{aligned}
$$

where $C 1$ is used to guarantee that SIC can be implemented successfully for a specific order. Constraint $C 2$ denotes the transmit power constraint for BS with the maximum power allowance $P_{\max }$. C3 represents the rate requirement of the $k$ th decoded user on subcarrier $n$. $C 4$ and $C 5$ denote that the number of users on each subcarrier cannot be more than $K_{f}$ and the number of subcarriers occupied by each user cannot be more than $N_{f}$.

The optimization problem (8) by jointly designing user, subcarrier assignment, and power allocation for the OFDM-NOMA system is a mixed-integer nonlinear programming(MINLP) problem, which generally yields unacceptable computation burden with brute-force search. Note that the optimization problem (8) includes the binary optimization variables for the user, subcarrier assignment, and the continuous variables for the power allocation coefficients. To solve problem (8) effectively, we will divide the problem into two subproblems: (1) the problem of user and subcarrier assignment and (2) the problem of power allocation among users.

\section{Resource Allocation}

3.1. User and Subcarrier Assignment Based on Different Speeds. In this section, the problem of user and subcarrier assignment based on different speeds will be solved. Assume that the power allocated to the users in each subcarrier is a fixed value, i.e., $P_{n}^{k}=P_{n}=P_{\max } / N, \forall n \in\{1, \cdots, N\}$. Thus, we can obtain the subproblem of user and subcarrier assign- ment as the following:

$$
\max _{\left\{\eta_{n, \pi(k)}\right\}} \varphi\left(\eta_{n}^{\pi(k)}\right)=\frac{R_{t o t}\left(\eta_{n}^{\pi(k)}\right)}{P_{t o t}\left(\eta_{n}^{\pi(k)}\right)},
$$

such that

$$
\begin{aligned}
C 1: g_{n}^{\pi(k)} \leq g_{n}^{\pi(j)}, \pi(k) \geq \pi(j), \forall n, k, \\
C 2: C_{n}^{\pi(k)} \geq R_{t h}, \forall n, k, \\
C 3: \sum_{k=1}^{K} \eta_{n, \pi(k)} \leq K_{f}, \\
C 4: \sum_{n=1}^{N} \eta_{n, \pi(k)} \leq N_{f}, \\
C 5: \eta_{n, \pi(k)}=\{0,1\}, \forall n, k .
\end{aligned}
$$

Note that the complexity of the brute-force search increases exponentially with the number of users and subcarriers, which makes it impractical. To maximize the sum rate, we consider the user and subcarrier assignment as a manyto-many matching process between the sets of users and subcarriers. To solve this problem, we adopt the matching theory, which provides mathematically tractable and lowcomplexity solutions for the combinatorial problem of matching players in two distinct sets $[16,17]$. We then propose an efficient algorithm to solve this problem.

3.2. Matching Theory for the User and Subcarrier Assignment. We design a low-complexity algorithm to solve the selection problem between users and subcarriers. First, we assume that the user set is $K, N$ is used to represent the subchannel set, and the two sets are disjoint. We treat the two sets as selfish and rational players with the aim of maximizing their respective interests. Each player can exchange information with each other without additional signaling cost because the channel state information (CSI) and speed are known to the BS. This means that each player has complete information about each other. If a subchannel $N_{j}$ is assigned to user $K_{i}$, then we say that $K_{i}$ and $N_{j}$ match each other, forming a matched pair. Matching is defined as pairing users in set $K$ with subcarriers in set $N$, formally expressed as follows:

Definition 1. Given two disjoint sets, $=\{1,2, \cdots, K\}$ of the users, and $\mathcal{N}=\{1,2, \cdots, N\}$ of the subchannels, a many-to many matching $\Psi$ is a mapping from the set $\mathscr{K} \cup \mathcal{N} \cup\{0\}$ into the set of all subsets of $\mathscr{K} \cup \mathcal{N}$ such that

(1) $|\Psi(k)| \leq l_{n}$ for every $k \in \mathscr{K}$

(2) $|\Psi(n)| \leq l_{k}$ for every $n \in \mathcal{N}$

(3) $\Psi(k) \in \mathscr{N}$ if and only if $\Psi(n) \in \mathscr{K}$

(4) $k=\Psi(n) \Leftrightarrow n=\Psi(k)$ 


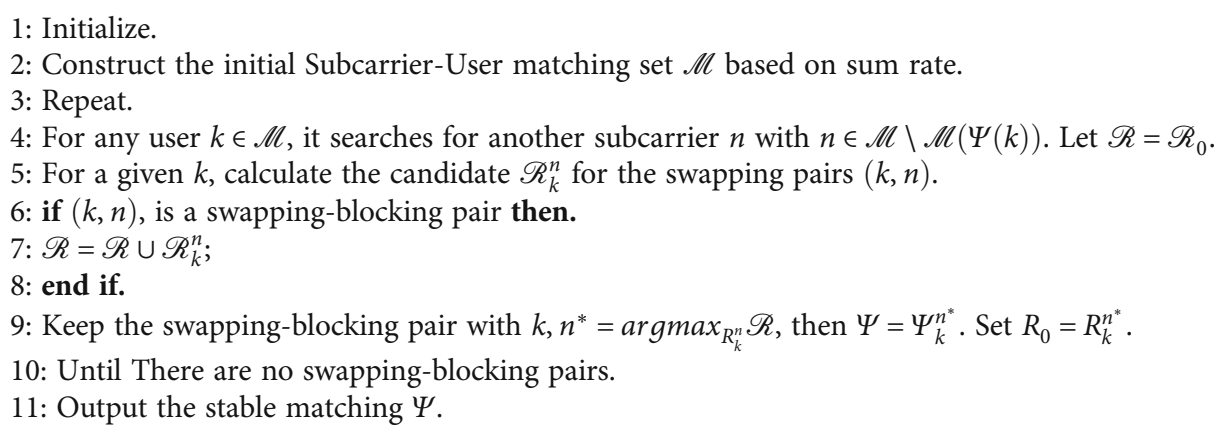

Algorithm 1: Many-to-many matching based SC-US assignment.

where $l_{n}$ and $l_{k}$ are positive integers. Condition (1) states that each user could occupy at most $l_{n}$ subchannels, and condition (2) implies that each subchannel can be allocated to at most $l_{k}$ users.

Then we introduce the conception of preference $>$ for both users and subchannels, to better describe the competition behavior and decision process of each player. For any $k_{i} \in \mathscr{K}$, if $k_{i}$ prefers $n_{1}$ to $n_{2}$, we express it as $n_{1}>_{k_{i}} n_{2}$.

It is not straightforward to define a stability concept in a many-to-many matching with externalities, as the gains from a matching pair depends on which players the other players have. However, a swap matching is defined as $\Psi_{i}^{j}=\{\Psi \backslash\{(i, k),(j, n)\} \cup\{(j, k),(i, n)\}\}$, where $\Psi(i)=k$ and $\Psi(j)=n$. Based on the swap operation, we introduce the two-sided exchange stability [18] as follows.

Definition 2. A matching $\Psi$ is two-sided exchange stable if and only if there does not exist a pair of players $(i, j)$ with $k=\Psi(i)$ and $n=\Psi(j)$, such that

(1) $\forall x \in\{i, j, k, n\}, \mathscr{U}_{k}\left(\Psi_{i}^{j}\right) \geq \mathscr{U}_{k}(\Psi)$

(2) $\exists x \in\{i, j, k, n\}$, such that $\mathscr{U}_{k}\left(\Psi_{i}^{j}\right) \geq \mathscr{U}_{k}(\Psi)$; then the swap matching $\Psi_{i}^{j}$ is approved, and $(i, j)$ is called a swap-blocking pair in $\Psi$

where $\mathscr{U}_{k}(\Psi)$ denotes the practicality for player $k$ under matching $\Psi$. In this paper, it means the rate of user $k$ in subcarrier $n$. In a nutshell, if any pair of players satisfied conditions (1) and (2), then it is called a swap-blocking pair.

The characteristics of the swap-blocking pair ensure that if the exchange match is approved, the achieved rate of any participating user will not decrease, and the rate of at least one user will increase.

As discussed above, the preference $>$ can be formulated as the rate of each user over all subcarriers. Specifically, the sum rate of users associated to subcarriers can be expressed as

$$
\mathscr{R}_{n}^{k}=\frac{B}{N} \log \left(1+r_{n}^{k}\right)
$$

Then the preference of subcarrier $n$ on a set of users $\Psi(k)$ can be expressed as $\mathscr{R}_{n}=\sum_{k \in \Psi(k)} \mathscr{R}_{n}^{k}$, and the preference of user $k$ occupying a set of subcarriers $\Psi(n)$ can be expressed as $\mathscr{R}^{k}=\sum_{n \in \Psi(n)} \mathscr{R}_{n}^{k}$.

Therefore, for a given user $k$ and any two subcarriers $n, n^{\prime}$, with two different matchings $\Psi$ and $\Psi^{\prime}$, we have

$$
(n, \Psi)>_{U E_{k}}\left(n^{\prime}, \Psi^{\prime}\right) \Leftrightarrow \mathscr{R}_{k}(\Psi)>\mathscr{R}_{k}\left(\Psi^{\prime}\right) .
$$

This means that user $k$ prefers subcarriers $n$ to $n^{\prime}$, because it can achieve a higher rate than subcarrier $n^{\prime}$. Similarly, for a given subcarrier $n$ and any two users $k, k^{\prime}$ with two different matchings $\Psi$ and $\Psi^{\prime}$, we have

$$
(k, \Psi)>_{S C_{n}}\left(k^{\prime}, \Psi^{\prime}\right) \Leftrightarrow \mathscr{R}^{n}(\Psi)>\mathscr{R}^{n}\left(\Psi^{\prime}\right)
$$

Then we introduce a matching Algorithm 1 to achieve matching.

3.3. Power Allocation for the Subcarrier. In the previous section, we solved the user and subcarrier assignment problem. We decompose the problem into subchannel power allocation and user power allocation within the same subchannel to obtain the suboptimal solution. In this section, we solve the subcarrier power allocation problem. So the problem can be converted as

$$
\begin{aligned}
& \max _{\left\{P_{n}^{k}\right\}} \varphi\left(P_{n}^{k}\right)=\frac{R_{t o t}\left(P_{n}^{k}\right)}{P_{\text {tot }}\left(P_{n}^{k}\right)}, \\
& C_{n}^{k}=\frac{B}{N} \log _{2}\left(1+r_{n}^{k}\right),
\end{aligned}
$$

such that

$$
\begin{aligned}
C 1: C_{n}^{k} & \geq R_{t h}, \forall n, k, \\
r_{n}^{k} & =\frac{\left|H_{n}^{k}\right|^{2} P_{n}^{k}}{\sum_{i=1}^{k-1}\left|H_{n}^{i}\right|^{2} P_{n}^{i}+\sum_{m=0, m \neq n}^{N-1} P_{m} \rho_{n, m}+\sigma_{\omega}^{2}} .
\end{aligned}
$$


Problem (11) is still a nonconvex problem due to $P_{n}$. Thus, to solve the problem efficiently, we consider a lower bound and a variable transformation, which was also used in $[19,20]$. Then we get a lower bound through the following inequality as follows.

$$
\log _{2}(1+\varsigma) \geq \Gamma \log _{2}(\bar{\varsigma}+\omega)
$$

with

$$
\Gamma=\frac{\bar{\varsigma}}{1+\bar{\varsigma}} \text {, and, } \omega=\log _{2}(1+\bar{\varsigma})-\bar{\varsigma} \log _{2} \frac{\bar{\varsigma}}{1+\bar{\varsigma}}
$$

where, if $\varsigma=\bar{\varsigma}$ at this time, the inequality becomes tight, and then $\log _{2}(1+\varsigma)=\Gamma \log _{2}(\bar{\varsigma}+\omega)$. Inspired by $(22)$ and as $\bar{\varsigma}$ corresponds to $r_{n}$, then we can obtain the following approximation of the lower bound:

$$
\underbrace{\sum_{n=1}^{N} \frac{B}{N} \log _{2} \Gamma\left(r_{n}^{k}+\omega\right)}_{\bar{\varphi}} \leq \varphi,
$$

where $\bar{\varphi}$ is the lower bound of (23), where $\Gamma=\left(r_{n}^{k}\right) /\left(1+r_{n}^{k}\right)$ and $\omega=\log _{2}\left(1+r_{n}^{k}\right)-r_{n}^{k} \log _{2}\left(\left(r_{n}^{k}\right) /\left(1+r_{n}^{k}\right)\right)$. When $\varsigma=\bar{\varsigma}$, we will have the following expression:

$$
\begin{aligned}
\frac{\sum_{n=1}^{N}(B / N) \log _{2} \Gamma\left(\left|H_{n}^{k}\right|^{2} P_{n}^{k}\right.}{P_{t o t}} & \frac{\left.\omega\left(\sum_{i=1}^{k-1}\left|H_{n}^{i}\right|^{2} P_{n}^{i}+\sum_{m=0, m \neq n}^{N-1} P_{m} \rho_{n, m}+\sigma_{\omega}^{2}\right)\right)}{P_{t o t}} \\
+ & -\frac{\sum_{n=1}^{N}(B / N) \log _{2} \Gamma\left(\sum_{i=1}^{k-1}\left|H_{n}^{i}\right|^{2} P_{n}^{i}+\sum_{m=0, m \neq n}^{N-1} P_{m} \rho_{n, m}+\sigma_{\omega}^{2}\right)}{P_{t o t}}
\end{aligned}
$$$$
\triangleq \bar{\varphi}
$$

Finally, we use this variable substitution $P_{n}^{k}=2^{\varepsilon_{n}^{k}}$, and then it becomes

$$
\begin{aligned}
\bar{\varphi}= & \frac{\sum_{n=1}^{N}(B / N) \log _{2} \Gamma\left(\left|H_{n}^{k}\right|^{2} 2^{\varepsilon_{n}^{k}}\right.}{P_{t o t}} \\
& +\frac{\left.\omega\left(\sum_{i=1}^{k-1}\left|H_{n}^{i}\right|^{2} 2^{\varepsilon_{n}^{i}}+\sum_{m=0, m \neq n}^{N-1} \sum_{k=1}^{K} 2^{\varepsilon_{n}^{k}} \rho_{n, m}+\sigma_{\omega}^{2}\right)\right)}{P_{t o t}} \\
& -\frac{\sum_{n=1}^{N}(B / N) \log _{2} \Gamma\left(\sum_{i=1}^{k-1}\left|H_{n}^{i}\right|^{2} 2^{\varepsilon_{n}^{i}}\right.}{P_{t o t}} \\
& +\frac{\left.\sum_{m=0, m \neq n}^{N-1} \sum_{k=1}^{K} 2^{\varepsilon_{n}^{k}} \rho_{n, m}+\sigma_{\omega}^{2}\right)}{P_{t o t}} \\
\triangleq & \frac{f\left(\varepsilon_{n}^{k}\right)}{g\left(\varepsilon_{n}^{k}\right)},
\end{aligned}
$$

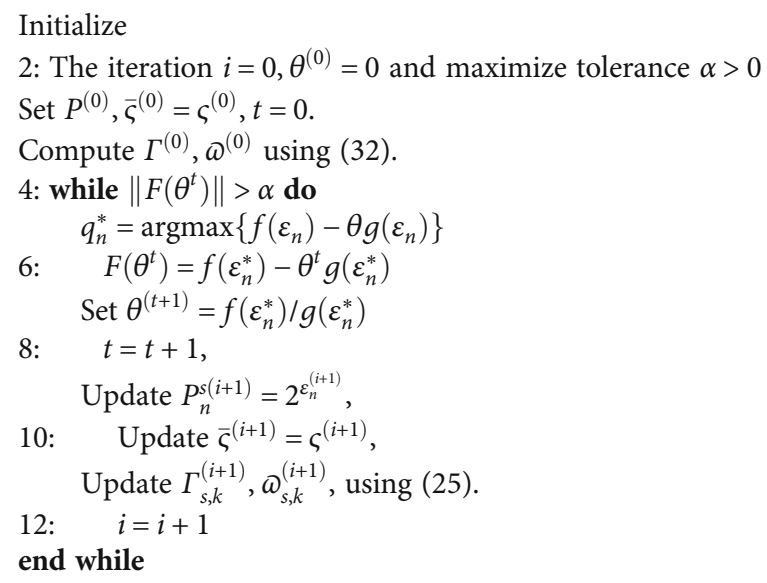

Algorithm 2: Power allocation for subchannel.

where $\varepsilon_{n}^{k}=\left[\varepsilon_{n}^{1}, \varepsilon_{n}^{2}, \cdots, \varepsilon_{n}^{k}, \cdots, \varepsilon_{N}^{K}\right]^{T}$ represent the power allocation vector for each user in each subchannel, and $(.)^{T}$ is denoted as the transpose operation. Then from(23), we can observe that $f\left(\varepsilon_{n}^{k}\right)$ is concave and $g\left(\varepsilon_{n}^{k}\right)$ is a convex due to the fact that the log-sum-exp function is convex [21, 22]. However, the user service quality (QOS) constraint $C_{n}^{k} \geq$ $R_{t h}$ can be expressed as

$$
\begin{aligned}
\left|H_{n}^{k}\right|^{2} 2^{\varepsilon_{n}^{k}} \geq & \left(2^{\frac{N}{B} R_{t h}}-1\right) \\
& \cdot\left(\sum_{i=1}^{k-1}\left|H_{n}^{i}\right|^{2} 2^{\varepsilon_{\mathrm{n}}^{i}}+\sum_{m=0, m \neq n}^{N-1} \sum_{k=1}^{K} 2^{\varepsilon_{n}^{k}} \rho_{n, m}+\sigma_{\omega}^{2}\right) .
\end{aligned}
$$

Then we apply the logarithmic operation at the both sides of (24), and we can obtain the expression of (25) as

$$
\begin{gathered}
\varepsilon_{n}^{k}+\log _{2}\left(\frac{\left|H_{n}^{k}\right|^{2}}{2^{(N / B) R_{t h}}-1}\right), \\
-\log _{2}\left(\sum_{i=1}^{k-1}\left|H_{n}^{i}\right|^{2} 2^{\varepsilon_{n}^{i}}+\sum_{m=0, m \neq n}^{N-1} \sum_{k=1}^{K} 2^{\varepsilon_{n}^{k}} \rho_{n, m}+\sigma_{\omega}^{2}\right) \geq 0 .
\end{gathered}
$$

Equation (25) is convex with respect to the variable set of $\varepsilon_{n}^{k}$. In order to obtain the power allocation vector of each user on each subcarrier, we first determine the power allocation budget $\varepsilon_{n}$ of each subcarrier, where $\varepsilon_{n}=\sum_{k=1}^{K_{f}} \varepsilon_{n}^{k}=P_{n}^{s}$. To this end, we deal with the following objective function.

$$
\max \bar{\varphi}\left(\varepsilon_{n}\right)=\frac{f\left(\varepsilon_{n}\right)}{g\left(\varepsilon_{n}\right)},
$$




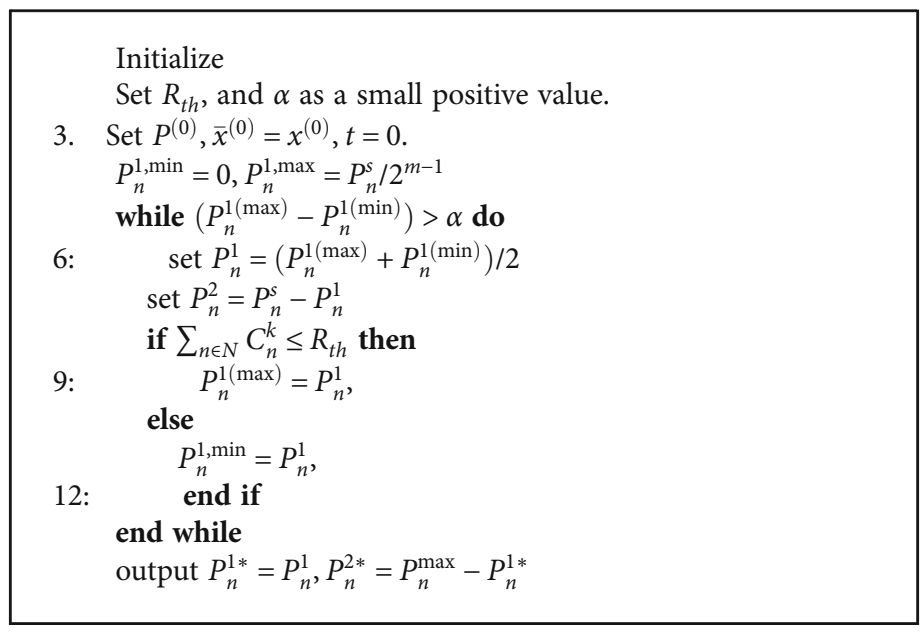

Algorithm 3: Power allocation between users on the same subchannel.

such that

$$
\begin{gathered}
C 1: \operatorname{in}(18), \\
C 4: \sum_{n=1}^{N} 2^{\varepsilon_{n}} \leq P_{\max }, \\
C 6: 2^{\varepsilon_{n}} \geq 0,
\end{gathered}
$$

where $\varepsilon_{n}=\left[\varepsilon_{1}, \varepsilon_{2}, \cdots, \varepsilon_{n}, \cdots, \varepsilon_{N}\right]^{T}$ represents the power budget of each subchannel $n$. Recalling that the log-sum-exp function is convex, it is clear that (28) has a concave numerator and a convex denominator for the objective function $\bar{u}$ over convex constraints [20]. In order to make the symbol more concise, we use $D$ to represent the domain of formula (18) to obtain a feasible solution to the optimization problem. Thus, let $\varepsilon_{n}^{*}$ and $\theta^{*}$ be the optimal solution and the maximum of (28), respectively. Then, we define the maximum $\bar{\varphi}, \theta^{*}$ of the considered system as

$$
\theta^{*}=\frac{f\left(\varepsilon_{n}^{*}\right)}{g\left(\varepsilon_{n}^{*}\right)}=\max _{\varepsilon_{n}} \frac{f\left(\varepsilon_{n}\right)}{g\left(\varepsilon_{n}\right)}, \forall \varepsilon_{n} \in D
$$

To this end, the following proposition is introduced.

Proposition 1. The maximum sum rate is achieved if and only if

$F\left(\theta^{*}\right)=\max _{\varepsilon_{n} \in D}\left\{f\left(\varepsilon_{n}\right)-\theta^{*} g\left(\varepsilon_{n}\right)\right\}=\max _{\varepsilon_{n} \in D}\left\{f\left(\varepsilon_{n}^{*}\right)-\theta^{*} g\left(\varepsilon_{n}^{*}\right)\right\}=0$.

Proof. We assume $q_{n}^{*}$ is the optimal solution to (28); then

$$
\theta^{*}=\frac{f\left(\varepsilon_{n}^{*}\right)}{g\left(\varepsilon_{n}^{*}\right)} \geq \frac{f\left(\varepsilon_{n}\right)}{g\left(\varepsilon_{n}\right)}, \forall \varepsilon_{n} \in D .
$$

This leads to

$$
\begin{aligned}
& f\left(\varepsilon_{n}\right)-\theta^{*} g\left(\varepsilon_{n}\right) \leq 0, \forall \varepsilon_{n} \in D, \\
& f\left(\varepsilon_{n}^{*}\right)-\theta^{*} g\left(\varepsilon_{n}^{*}\right)=0, \forall \varepsilon_{n} \in D .
\end{aligned}
$$

Therefore, $\varepsilon_{n}^{*}$ satisfies (31) and $F\left(\theta^{*}\right)=0$.

Conversely, let $\varepsilon_{n}^{*}=\operatorname{argmax}\left\{f\left(\varepsilon_{n}\right)-\theta^{*} g\left(\varepsilon_{n}\right)\right\}$. This implies

$$
f\left(\varepsilon_{n}\right)-\theta^{*} g\left(\varepsilon_{n}\right) \leq f\left(\varepsilon_{n}^{*}\right)-\theta^{*} g\left(\varepsilon_{n}^{*}\right)=F\left(\theta^{*}\right)=0, \forall \varepsilon_{n} \in D,
$$

in which the two conditions can be rewritten as

$$
\begin{aligned}
& \theta^{*} \geq \frac{f\left(\varepsilon_{n}\right)}{g\left(\varepsilon_{n}\right)}, \forall q_{n} \in D, \\
& \theta^{*}=\frac{f\left(\varepsilon_{n}^{*}\right)}{g\left(\varepsilon_{n}^{*}\right)} .
\end{aligned}
$$

Thus, the proof is completed.

So, we can find the optimal solutions for fractional problem (28) by finding the unique zero of (30), i.e., the auxiliary function $F(\theta)=f\left(\varepsilon_{n}\right)-\delta g\left(\varepsilon_{n}\right)$.

Therefore, we solve (11), and its equivalent objective function is (26), which can be achieved by designing an iterative algorithm. The algorithm is based on Dinkelbach's algorithm, which is summarized as Algorithm 1. Given the $t$ value of $\theta^{t}$ in each iteration, we calculate the power allocation $\varepsilon_{n}^{t}$ by solving the equivalent problem (28). Then we obtain $\varepsilon_{n}^{t}$ by updating $\theta^{(t+1)}=\left(f\left(\varepsilon_{n}^{(t)}\right)\right) /\left(g\left(\varepsilon_{n}^{(t)}\right)\right)$. We pay attention to the Dinkelbach method based on Newton's 


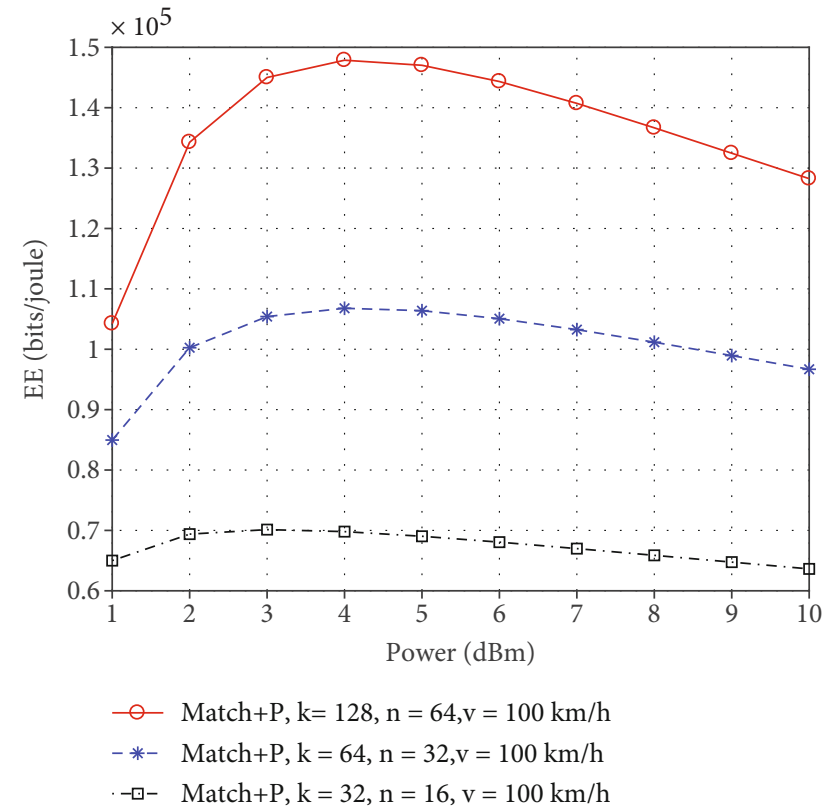

Figure 2: EE of different subcarriers and users at different powers.

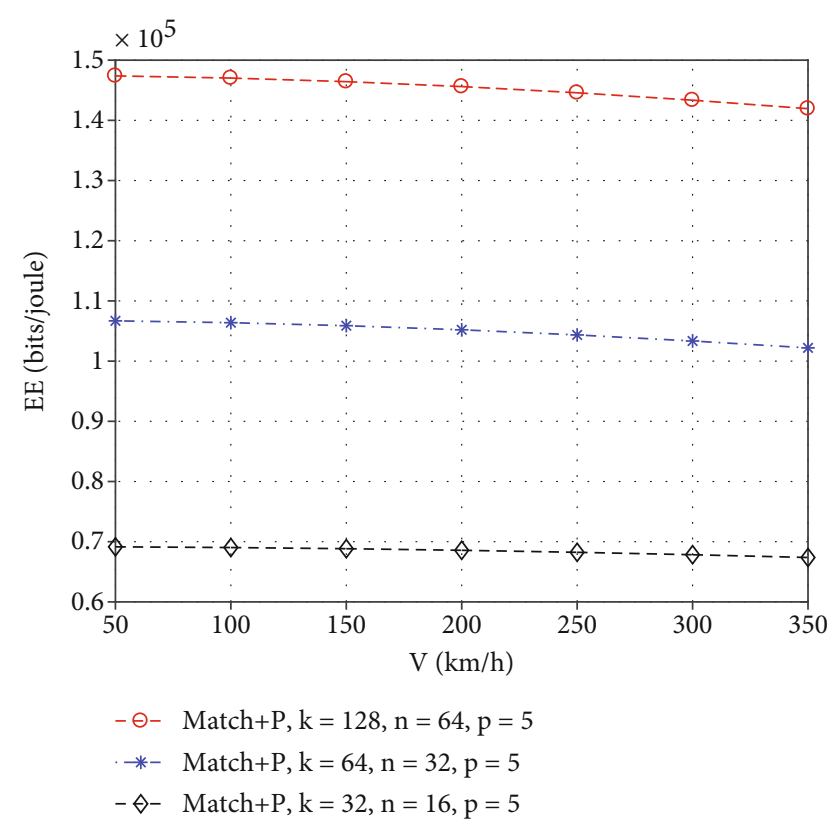

Figure 3: EE of different subcarriers and users at different speeds.

method in fractional programming since the update can be obtained in the following way:

$$
\theta^{(t+1)}=\theta^{(t)}-\frac{F\left(\theta^{(t)}\right)}{F^{\prime}\left(\theta^{(t)}\right)}=\frac{f\left(\varepsilon_{n}^{(t)}\right)}{g\left(\varepsilon_{n}^{(t)}\right)} .
$$

Therefore, as long as (28) is solved in each iteration, the iterative algorithm can guarantee to converge to the

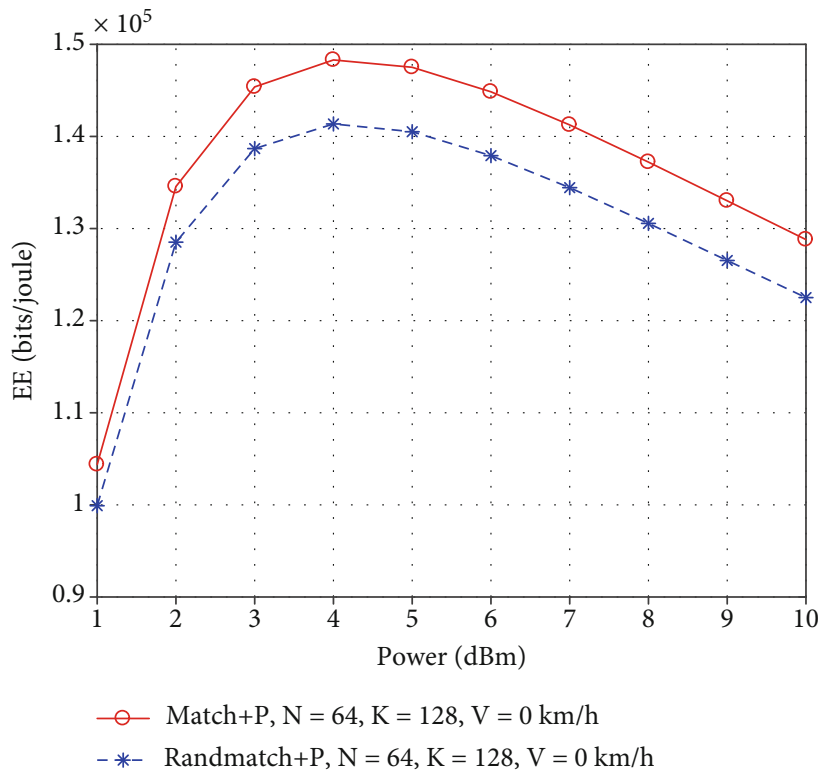

FIgURE 4: EE and power under different matching algorithms.

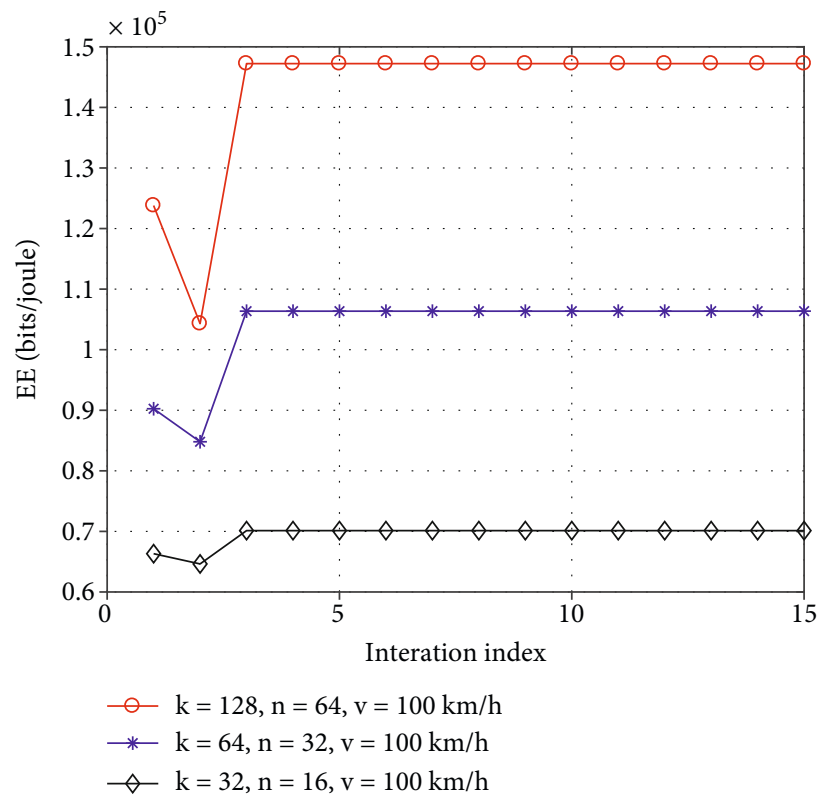

FIGURE 5: Iterative convergence of EE and algorithm for different subcarriers and users.

optimal value $\theta^{*}$. The proposed algorithm is presented in Algorithm 2.

Through Algorithm 2, we solve the problem of power allocation between subchannels, so we further solve the problem of power allocation among users of the same subchannel. At this point, we transform the problem into

$$
\max \bar{\varphi}\left(\varepsilon_{n}^{k}\right)=\frac{f\left(\varepsilon_{n}^{k}\right)}{g\left(\varepsilon_{n}^{k}\right)}
$$


TABle 1: Comparison of various algorithms.

\begin{tabular}{lcc}
\hline Algorithms & Complexity & Optimality \\
\hline Match+P & $\mathcal{O}\left(\left(K+N^{2}\right)+\left(V K+V^{\prime} K_{f} N_{f} N\right)+\mathcal{O}(\bar{V} N)\right.$ & Suboptimal \\
Randmatch+P & $\mathcal{O}\left(\left(K+N^{2}\right)+\mathcal{O}(\bar{V} N)\right.$ & Suboptimal \\
Match+SCA [26] & $\mathcal{O}\left(\left(K+N^{2}\right)+\left(V K+V^{\prime} K_{f} N_{f} N\right)+\mathcal{O}(\bar{V}(N K))^{3}\right)$ & Suboptimal \\
\hline
\end{tabular}

such that

$$
\begin{gathered}
C 1: C_{n}^{k} \geq R_{t h}, \forall n, k, \\
C 4: \sum_{n=1}^{N} \sum_{k=1}^{K} 2^{\varepsilon_{n}^{k}} \leq P_{\max }, \\
C 6: 2^{\varepsilon_{n}^{k}} \geq 0 .
\end{gathered}
$$

Obviously, for the power allocation among users of the same subchannel, the power allocated by each subchannel cannot exceed the power allocation budget $P_{n}^{s}$. According to the theory in $[23,24]$, for a user with a strong channel gain, his assignable power is between 0 and $P_{n}^{s} / 2^{m-1}$, where $m$ is the number of users allocated in the same subchannel and $P_{n}^{s}$ is the power budget of each subchannel $n$ [25]. We assume that at most $K_{n}$ users are multiplexed on the same subchannel; in order to reduce the complexity of the receiver, we assume that each subchannel carries at most 2 users, that is, $K_{n}=2$. Then we use a binary search algorithm to solve the power allocation problem for users on the same subchannel, which was given in Algorithm 3.

\section{Simulation}

In this part, we analyze the simulation performance of the system model; in Figure 2, we, respectively, set $K=128$, $N=64$, and $V=100 \mathrm{~km} / \mathrm{h} ; K=64, N=32$, and $V=100 \mathrm{~km} / \mathrm{h}$; and $K=32, N=16$, and $V=100 \mathrm{~km} / \mathrm{h}$. It can be seen from the simulation results of Figure 2 that as the power increases, EE first increases and then decreases. It shows that within a certain power range, the system model has an optimal power distribution situation.

Figure 3 reflects the EE at different speeds for different subcarriers and users. From the figure, we can see that with the increase of user speed, EE gradually decreases; especially when the number of users and the number of subcarriers are large, the effect is more obvious. This is because as the number of subcarriers increases, the spacing between carriers becomes smaller, and the greater the total ICI generated by the system. Figure 4 presents the comparison of random matching plus power distribution and matching plus power distribution under EE conditions at zero speed and different powers. It can be seen that the proposed algorithm has a significant improvement on EE.

Figure 5 shows the relationship between the convergence of the proposed algorithm and EE. It can be seen from Figure 5 that when the number of iterations reaches 3 , the algorithm has completely converged.
Table 1 shows the performance comparison of various algorithms, where $V$ and $V^{\prime}$ represent the number of iterations of Algorithms 2 and 3. As can be seen from the table, the algorithm proposed in this paper has higher computational complexity than random matching, but it has better EE. Compared with SCA, the proposed algorithm has relatively low computational complexity and can obtain approximate performance.

Reference [24] does not consider the impact of ICI on EE generated by users at different speeds. Compared with reference [26], although the branch and bound (BB) approach can be used to obtain the optimal solution, it will produce unacceptable computational complexity. The algorithm proposed in this paper can only obtain suboptimal solutions, but it can greatly reduce the calculation time.

\section{Conclusion}

In this paper, we mainly study the resource allocation of the OFDM-NOMA system in multiuser scenarios with different speeds. The resource allocation problem we describe is NPhard, so we decompose the resource allocation problem into two subproblems for solving, namely the BS-SC matching problem and the power allocation problem. First, BS-SC is matched according to the matching theory, and then power allocation is carried out for the BS-SC pairs successfully matched. Two suboptimal algorithms are proposed to solve the nonconvex optimization problem.

\section{Data Availability}

The data used to support the findings of this study are included within the article.

\section{Conflicts of Interest}

The authors declare that they have no conflicts of interest.

\section{Acknowledgments}

This work was supported in part by the National Science Foundation of China under grant 61561046, in part by Key Research \& Development and Transformation Plan of Science and Technology Program for Tibet Autonomous Region (No. XZ201901-GB-16), in part by the Tibet University Special Fund Project for the Reform and Development of Local Colleges and Universities Supported by the Central Government in 2021 (The First-Class Discipline Cultivation Project), in part by the Central Government that supports 
the reform and development of local universities of Tibet University in 2020, in part by the Special Fund for the Development of Local Universities supported by the Central Finance of Tibet University in 2019.

\section{References}

[1] V. W. Wong, Key Technologies for $5 G$ Wireless Systems, Cambridge university press, 2017.

[2] Y. Liu, Z. Qin, M. Elkashlan, Z. Ding, A. Nallanathan, and L. Hanzo, "Non-orthogonal multiple access for $5 \mathrm{G}$ and beyond," Proceedings of the IEEE, vol. 105, no. 12, pp. 23472381, 2017.

[3] A. Benjebbour, Y. Saito, Y. Kishiyama, A. Li, A. Harada, and T. Nakamura, "Concept and practical considerations of nonorthogonal multiple access (NOMA) for future radio access," in 2013 International Symposium on Intelligent Signal Processing and Communication Systems, Naha, Japan, 2013.

[4] W. Liang, Z. Ding, Y. Li, and L. Song, "User pairing for downlink non-orthogonal multiple access networks using matching algorithm," IEEE Transactions on Communications, vol. 65, pp. 5319-5332, 2017.

[5] B. Di, S. Bayat, L. Song, and Y. Li, "Radio resource allocation for downlink non-orthogonal multiple access (NOMA) networks using matching theory," in 2015 IEEE global communications conference (GLOBECOM), San Diego, CA, USA, 2015.

[6] F. Fang, J. Cheng, and Z. Ding, "Joint energy efficient subchannel and power optimization for a downlink NOMA heterogeneous network," IEEE Transactions on Vehicular Technology, vol. 68, no. 2, pp. 1351-1364, 2019.

[7] H. Zhang, M. Feng, K. Long, G. K. Karagiannidis, V. C. Leung, and H. V. Poor, "Energy efficient resource management in SWIPT enabled heterogeneous networks with NOMA," IEEE Transactions on Wireless Communications, vol. 19, pp. 835845, 2019.

[8] X. Song, L. Dong, J. Wang, L. Qin, and X. Han, "Energy efficient power allocation for downlink NOMA heterogeneous networks with imperfect CSI," IEEE Access, vol. 7, pp. 3932939340, 2019.

[9] C. Xu, G. Zheng, and L. Tang, "Energy-aware user association for NOMA-based mobile edge computing using matchingcoalition game," IEEE Access, vol. 8, pp. 61943-61955, 2020.

[10] M. R. Zamani, M. Eslami, M. Khorramizadeh, H. Zamani, and Z. Ding, "Optimizing weighted-sum energy efficiency in downlink and uplink NOMA systems," IEEE Transactions on Vehicular Technology, vol. 69, pp. 11112-11127, 2020.

[11] W. Zhao, R. She, and H. Bao, "Security energy efficiency maximization for two-way relay assisted cognitive radio NOMA network with self-interference harvesting," IEEE Access, vol. 7, pp. 74401-74411, 2019.

[12] C. Yang-Seok, P. J. Voltz, and F. A. Cassara, "On channel estimation and detection for multicarrier signals in fast and selective Rayleigh fading channels," IEEE Transactions on Communications, vol. 49, no. 8, pp. 1375-1387, 2001.

[13] M. Russell and G. L. Stuber, "Interchannel interference analysis of OFDM in a mobile environment," in 1995 IEEE 45th vehicular technology conference. Countdown to the Wireless Twenty-First Century, vol. 2, pp. 820-824, Chicago, IL, USA, 1995.

[14] Z. Dong, P. Fan, and E. Panayirci, "Effect of power and rate adaptation on the spectral efficiency of MQAM/OFDM system under very fast fading channels," Eurasip Journal on Wireless Communications Networking, vol. 1, no. 2012, p. 208, 2012.

[15] H. Cheon and D. Hong, "Effect of channel estimation error in OFDM-based WLAN," IEEE Communications Letters, vol. 6, no. 5, pp. 190-192, 2002.

[16] Y. Gu, Y. Zhang, M. Pan, and Z. Han, "Matching and cheating in device to device communications underlying cellular networks," IEEE Journal on Selected Areas in Communications, vol. 33, no. 10, pp. 2156-2166, 2015.

[17] J. Cui, Y. Liu, Z. Ding, P. Fan, and A. Nallanathan, “Optimal user scheduling and power allocation for millimeter wave NOMA systems," IEEE Transactions on Wireless Communications, vol. 17, no. 3, pp. 1502-1517, 2018.

[18] E. Bodine-Baron, C. Lee, A. Chong, B. Hassibi, and A. Wierman, "Peer effects and stability in matching markets," in Proc. Int. Symp. Algorithmic Game Theory, pp. 117-129, Springer, Berlin, Germany, 2011.

[19] J. Papandriopoulos and J. S. Evans, "Low-complexity distributed algorithms for spectrum balancing in multi-user DSL networks," in 2006 IEEE International Conference on Communications, Istanbul, Turkey, 2006.

[20] A. Zappone and E. Jorswieck, "Energy efficiency in wireless networks via fractional programming theory," Foundations Trends in Communications Information Theory, vol. 11, no. 3-4, pp. 185-396, 2015.

[21] A. Zappone and E. A. Jorswieck, "Energy-efficient resource allocation in future wireless networks by sequential fractional programming," Digital Signal Processing, vol. 60, pp. 324337, 2017.

[22] S. Boyd, S. P. Boyd, and L. Vandenberghe, Convex Optimization, Cambridge university press, 2004.

[23] M. S. Ali, H. Tabassum, and E. Hossain, "Dynamic user clustering and power allocation for uplink and downlink non-orthogonal multiple access (NOMA) systems," IEEE Access, vol. 4, pp. 6325-6343, 2016.

[24] A. J. Muhammed, Z. Ma, P. D. Diamantoulakis, L. Li, and G. K. Karagiannidis, "Energy-efficient resource allocation in multicarrier NOMA systems with fairness," IEEE Transactions on Communications, vol. 67, no. 12, pp. 8639-8654, 2019.

[25] M. S. Ali, E. Hossain, and D. I. Kim, "Coordinated multipoint transmission in downlink multi-cell NOMA systems: models and spectral efficiency performance," IEEE Wireless Communications, vol. 25, no. 2, pp. 24-31, 2018.

[26] J. Cui, Y. Liu, Z. Ding, P. Fan, and A. Nallanathan, "QoE-based resource allocation for multi-cell NOMA networks," IEEE Transactions on Wireless Communications, vol. 17, no. 9, pp. 6160-6176, 2018. 